\title{
TWO EXTREME DIOPHANTINE PROBLEMS CONCERNING THE PERIMETER OF PYTHAGOREAN TRIANGLES
}

\author{
Allan J. MacLeod
}

University of the West of Scotland, U.K.

\begin{abstract}
We consider two Diophantine problems involving the perimeter of Pythagorean triangles. One has an enormous solution with the sides of the triangle $>10^{115}$, whilst the other has no solution which answers a speculation of Frenicle de Bressy.
\end{abstract}

\section{INTRODUCTION}

There are a large number of Diophantine problems, related to the properties of a right-angled triangle with integer sides, mentioned in Book VI of Diophantus ([2]) and Chapter 4 of Dickson ([1]). Some of these involve the area and perimeter. We consider one problem explicitly mentioned and another problem which is a natural extension of one discussed by both Fermat and Frenicle de Bressy.

\section{Problem One}

On page 178, Dickson states

Fermat noted that if in $(205769,190281,78320)$ we add the area to the square of the sum of the legs, we get a square.

Frenicle stated the last result without comment; also that the sum of the area and hypotenuse of $(17,144,145)$ is a square; while the first three right triangles in which the sum of the area and smaller leg is a square are $(3,4,5)$, $(16,30,34),(105,208,233)$.

2010 Mathematics Subject Classification. 01A45, 11-03, 11D09, 11 Y50.

Key words and phrases. Right-angled triangle, perimeter, elliptic curve. 
It should be noted that only the first problem makes real sense, since the area and legs are measured in different units. Thus only by squaring the lengths do we get a problem which is independent of the units of measurement.

Not mentioned in this section is the natural problem of finding a triangle where the area plus the square of the perimeter is a square.

We can, without loss of generality, assume the legs are $a=2 p q$ and $b=p^{2}-q^{2}$, with hypotenuse $h=p^{2}+q^{2}$, where $p, q$ are positive integers of opposite parity with no common factor with $p>q$.

Thus we require an integer $d$ such that

$$
d^{2}=(p q)\left(p^{2}-q^{2}\right)+\left(2 p^{2}+2 p q\right)^{2}=4 p^{4}+9 p^{3} q+4 p^{2} q^{2}-p q^{3} .
$$

This quartic clearly has a solution at $p=1, q=1, d=4$, so is birationally equivalent to an elliptic curve. Using Mordell ([3]), we find this elliptic curve to be

$$
E: y^{2}=x^{3}+7 x^{2}+2 x
$$

with the transformation

$$
\frac{p}{q}=\frac{9 x-4 y+1}{1-16 x} .
$$

The elliptic curve $E$ has the obvious point of order 2 at $T=(0,0)$. The other zeroes of the curve are at $-3.5 \pm \sqrt{10.25}$, so the elliptic curve consists of 2 components. The first is usually called the "egg" and is a finite closed curve which exists for (approximately) $-6.70156 \leq x \leq-0.29844$, while the second is an infinite component for $x \geq 0$.

There are no points of order 4 , as this would require the linear coefficient to be a rational square, which 2 is not. For points of order 3, we investigate the points of inflexion which satisfy

$$
3 x^{4}+28 x^{3}+12 x^{2}-4=0
$$

which has no integer roots - the Nagell-Lutz theorem ensures that any torsion points have integer coordinates.

Thus the torsion subgroup (points of finite order) of the Abelian group of rational points is isomorphic to $\mathbb{Z}_{2}$. The conductor is 1312 , and using the online version of Cremona's Elliptic Curve Tables, we find the curve to be designated $1312 B 1$, which has rank 1 with generator $G=(-2,4)$. Thus all rational points on $E$ are of the form $n G, n G+T, n G-T$ for $n \in \mathbb{Z}$. Note that, if $P=(u, v)$ then $P+T=\left(2 / u,-2 v / u^{2}\right)$.

This set of points can be simplified due to the following facts:

- Since $T=-T$, we get the same $p / q$ ratio from adding either.

- Numerical experiments show that $(-n+1) G$ gives the same value of $p / q$ as $(n+1) G$. This can be proven by standard algebraic manipulation.

- If a point $P$ gives $p / q=r$ then $P+T$ gives $p / q=(r+1) /(r-1)$, which gives the triangle with sides $(2 b, 2 a, 2 h)$. 
Thus, we need only investigate points of the form $n G$ for $n \geq 1$. For the point $G, p / q=-1$ which is not acceptable. $2 G=(1 / 16,-25 / 64)$ which gives an undefined value of $p / q .3 G=(-578 / 1089,-31348 / 35937)$ which gives $p / q=-1 / 33$, again unacceptable, and we find this for $4 G, 5 G, \ldots, 9 G$.

The reason can be found from the requirement that real-world triangles need $p / q>1$. Thus

$$
\frac{4 y-25 x}{16 x-1}>0
$$

Thus, if $x>1 / 16$, we need $y>25 x / 4$, but, if $x<1 / 16$, we must have $y<25 x / 4$.

Thus we need to know where the line $y=25 x / 4$ meets the curve. The intersections occur when

$$
16 x^{3}-513 x^{2}+32 x=0=x(16 x-1)(x-32),
$$

so $x=0,1 / 16,32$. A consideration of the geometry shows we need $0<x<$ $1 / 16$ or $x>32$, for $p / q>1$. The fact that the 3 points of intersection satisfy $x \geq 0$ means that the entire egg must lie totally above or totally below the line, and it is easy to see that the egg is above, so contributes no points that could provide a solution.

Both the generator $G$ and the alternative $G+T$ lie on the egg. Any point $2 Q$ has a square $x$-coordinate so lies on the infinite part. Thus $(2 k+1) G$ and $(2 k+1) G+T$ lie on the egg, so half the rational points are ruled out automatically as possible solutions.

Eventually, we had to use a package such as Pari/GP to do the arithmetic. In fact $p / q>1$ only when we reached $n=14$. We find $14 G$ gives $p / q \approx 4.37$ with

$$
\begin{aligned}
& p=13429113308414333597592845284336853930977325063518524585889 \\
& q=3075204171617894263584790454415418855508600241802333917600
\end{aligned}
$$

which gives a triangle with

$$
\begin{aligned}
& a=8259453053433028030760467401818982186863625970140904562357 \\
& 9749623226194029676793767412433328848114540834478097492800,
\end{aligned}
$$$$
b=1708842035530948692483352277209108546409113692277871586712
$$$$
37720545012463595324309972334647278599491419653995348160321 \text {, }
$$$$
h=189797964947367067797550498433169961371645505457029686258
$$$$
068466010450419601182263515365657729302307416716722527680321 .
$$

We find that acceptable triangles occur when $n=14,28,42,54,68,82,94, \ldots$ 


\section{Problem Two}

Towards the end of Chapter IV of Dickson is the statement

Frenicle noted that if the hypotenuse and perimeter of a right triangle are squares, the perimeter has at least 13 digits.

The implication is that Frenicle considered the problem and could not find a small solution.

Thus $h=p^{2}+q^{2}=r^{2}$ and $2 p^{2}+2 p q=s^{2}$. We have $(p, q, r)$ forming a Pythagorean triple, so $p=u^{2}-v^{2}, q=2 u v$, since we can obviously ignore any multiplier.

Thus,

(3.1) $s^{2}=2\left(u^{2}-v^{2}\right)^{2}+2\left(u^{2}-v^{2}\right) 2 u v=2 u^{4}+4 u^{3} v-4 u^{2} v^{2}-4 u v^{3}+2 v^{4}$.

Defining $y=s / v^{2}, x=u / v$, we have the quartic

$$
y^{2}=2 x^{4}+4 x^{3}-4 x^{2}-4 x+2,
$$

which has the solution $x=1, y=0$. Thus the quartic is birationally equivalent to an elliptic curve.

Using the transformations $x=(k+1) /(k-1)$ and $y=4 w /(k-1)^{2}$ we derive the elliptic curve

$$
w^{2}=k^{3}+2 k^{2}-k .
$$

This has conductor 128 and the curve is $128 D 2$ in Cremona's Table. This has rank 0 and only 1 finite torsion point $(0,0)$.

Thus there can be no solution to the original problem. If, however, we change perimeter to semi-perimeter, we find the following.

The quartic changes to

$$
s^{2}=\left(u^{2}-v^{2}\right)^{2}+\left(u^{2}-v^{2}\right) 2 u v=u^{4}+2 u^{3} v-2 u^{2} v^{2}-2 u v^{3}+v^{4}
$$

and this is birationally equivalent to the elliptic curve

$$
y^{2}=x^{3}+4 x^{2}-4 x
$$

via the transformation

$$
\frac{u}{v}=\frac{x-y}{2-2 x}
$$

This gives the relation

$$
\frac{p}{q}=\frac{x^{3}+x^{2}+2 x(2-y)-4}{4(1-x)(x-y)} .
$$

The elliptic curve is $128 A 2$ in Cremona's Tables with one finite torsion points at $T=(0,0)$, and rank 1 with generator $H=(-2,4)$.

We compute $n H$ and $n H+T$ for $n=1,2,3, \ldots$, until $p / q>1$. This occurs for $n=5$, and leads to the triangle with $a=12144836581416, b=$ 4599272822087 and $h=12986545579225$, all of which have at least 13 digits. So the Frenicle statement is true if we replace perimeter by semi-perimeter. 


\section{ACKNOWLEDGEMENTS}

The author would like to thank the editor and, especially, an unknown referee for several very helpful comments which have greatly improved the manuscript.

\section{REFERENCES}

[1] L. E. Dickson, History of the theory of numbers, Vol. II. Diophantine analysis, AMS Chelsea Publishing co., New York, 1992.

[2] T. L. Heath, Diophantus of Alexandria: A Study in the history of Greek algebra, Cambridge University Press, Cambridge, 1910.

[3] L. J. Mordell, Diophantine Equations, Academic Press, London-New York, 1969.

A. J. MacLeod

Statistics and Mathematics Group

University of the West of Scotland

High St. Paisley

Scotland PA1 2BE

E-mail: allan.macleod@uws.ac.uk

Received: 7.1.2010.

Revised: 11.2 .2010 . 